EUROPEAN JOURNAL OF PURE AND APPLIED MATHEMATICS

Vol. 11, No. 1, 2018, 169-188

ISSN 1307-5543 - www.ejpam.com

Published by New York Business Global

\title{
On Prime Hyperfilters (Hyperideals) in $\bigwedge$-Hyperlattices
}

\author{
M. Amiri Bideshki ${ }^{1}$, R. Ameri ${ }^{2}, *$, A. Borumand Saeid ${ }^{3}$ \\ ${ }^{1}$ Department of Mathematics, Farhangian University, Kerman, Iran \\ 2 School of Mathematics, Statistic and Computer Sciences, University of Tehran, Tehran, \\ Iran \\ ${ }^{3}$ Department of Mathematics, Shahid Bahonar University, Kerman, Iran
}

\begin{abstract}
In this paper, we introduce the notions of strong " $\wedge$ "-hyperlattices, hyperideals and hyperfilters in strong " $\wedge$ "-hyperlattices. Also, we give equivalence conditions for prime hyperfilters (hyperideal) in strong $\wedge$-hyperlattices. Distributivity (dual distributivity) in " $\wedge$ "-hyperlattices,$I_{A^{-}}$ hyperideals and prime hyperfilters in strong " $\wedge$ "-hyperlattices are investigated.
\end{abstract}

2010 Mathematics Subject Classifications: 20N20

Key Words and Phrases: Hyperideal, Hyperfilter, Hyperlattice, Strong $\wedge$ hyperlattice, $I_{A^{-}}$ Hyperideal

\section{Introduction}

Theory of hyperalgebra has been introduced by F. Marty in the eighth congress of Scandinavians in 1934 [11]. Several aspects of subalgebra and subdirect decompositions of hyperalgebra were studied by Pickett and by Hansoul, for more details see [5], [17], and [18]. In [22] congruence of multialgebra has been studied by D. Schweigrt. In[3] Ameri and M. M. Zahedi introduced and studied notion of hyperalgebraic systems. In[1] Ameri and Nozari Studied relationship between the categories of multialgebra and algebra; In [2] Ameri and Rosenberg studied congruences and strongly congruences of multialgebras. Some more basic properties of hyperalgebra such as, identities, term function and fundamental relation, direct limit of hyperalgebra, and the exponentiation of universal hyperalgebra have been studied by C. Pelea and others, for more details see [13], [14], and [15]. In[16] C. Pelea and I. Purdea have been proved that complete hyperalgebra can be obtained from a universal algebra and a appropriate congruence on it.

Theory of hyperlattices introduced by Konstantinidou and J. Mittas in 1977 [10]. M. Konstantinidou, also studied distributive, modular, and complemented hyperlattices, for more details see [8] and [9]. In [19] Rahnemaei Barghi considered the prime ideal theorem for

${ }^{*}$ Corresponding author.

Email addresses: Amirimohsen61@gmail.com (M. Amiri Bideshki), R. Ameri@ut.ac.ir (R. Ameri), Arsham@uk.ac.ir (A. Borumand Saeid) 
distributive hyperlattices. In [12], G. A. Moghani and A. R. Ashrafi proved that in some cases the set of all subhypergroups $\mathrm{G}$ has a hyperlattice structure. In [23], X. L. Xin and X. G. Li studied hyperlattices and quotient hyperlattices. In [4], A. Asokkumar proved that under certain conditions, the idempotent elements of a hyperring form a hyperlattice and the orthogonal idempotent elements form a quassi-distributive hyperboolean algebra. In [7], B. B. N. Koguep, C. Nkuimi, and C. Lele studied ideals and filters in hyperlattices. Rasouli and Davvaz defined fundemental relation on a hyperlattice and obtained a lattice from a hyperlattice; Also they defined a topology on the set of prime ideals of a distributive hyperlattice, see [20] and [21]. P. He, X. Xin and Jianming Zhan studied and introduced rough hyperideal in a hyperlattice, also they investigated some properties about homomorphic images of rough hyperideals in hyperlattices [6]. In section 2, we study $" \wedge "$-hyperlattice and some properties of it. Also in this section distributivity and dual distributivity in $\wedge$-hyperlattices are studied. In section 3 , we investigate hyperideals and hyperfilters in $\wedge$-hyperlattice $L$ and we prove that $(P \wedge F(a)) \vee(P \wedge F(b))=P \wedge F(a \vee b)$, where $P$ is a hyperfilter and $F(a)$ is the generating filter by $a \in L$. In section 4 , we introduce $I_{A}$-hyperideal, for some non-empty subset $A$ of a dual distributive " $\wedge$ "-hyperlattice $L$; Also In this section, " $\wedge$ "subhyperlattices are studied.

\section{Preliminaries}

Before we study $\wedge$-hyperlattice and some result of it, let us state some terminologies.

Definition 1. [11] Let $H$ be a non-empty set and $P^{*}(H)$ denotes the set of all nonempty subsets of $H$. Maps of the following type:

$$
f: H \times H \longrightarrow P^{*}(H),
$$

are called hyper operation and $(H, f)$ that satisfies some axioms is called a hyperalgebra.

Definition 2. [10] Let $L$ be a nonempty set, $\wedge-$ be a binary operation, and $\vee-$ be a hyper operation on $L$. Then $L$ is called a hyperlattice, if for all $a, b, c \in L$, the following conditions hold:

(i) $a \in a \vee a$, and $a \wedge a=a$;

(ii) $a \vee b=b \vee a$, and $a \wedge b=b \wedge a$;

(iii) $a \in[a \wedge(a \vee b)] \cap[a \vee(a \wedge b)]$;

(iv) $a \vee(b \vee c)=(a \vee b) \vee c$, and $a \wedge(b \wedge c)=(a \wedge b) \wedge c$;

(v) $a \in a \vee b \Longrightarrow a \wedge b=b$.

Definition 3. [9] A hyperlattice $L$ is called bounded if there exist $0,1 \in L$ such that for all $x \in L, 0 \leq x \leq 1$. 
M. Amiri Bideshki, R. Ameri, A. Borumand Saeid / Eur. J. Pure Appl. Math, 11 (1) (2018), 169-188 171

Let $(L, \oplus, \otimes)$ be a hyperalgebra and $A \subseteq L$. We say that $A$ is $\oplus$-Closed, if $a \oplus b \subseteq A$, for all $a, b \in A$.

Definition 4. Let $L$ be a nonempty set, " $\wedge$ " and " $\vee$ " are hyperoperation and binary operation, respectively. Tnen $L$ is called $a " \wedge "-$ hyperlattice if

(i) $a \in a \wedge a, a \vee a=a$,

(ii) $a \wedge b=b \wedge a, a \vee b=b \vee a$,

(iii) $a \wedge(b \wedge c)=(a \wedge b) \wedge c, a \vee(b \vee c)=(a \vee b) \vee c$,

(iv) $a \in(a \wedge(a \vee b)) \cap(a \vee(a \wedge b))$,

and $L$ is called strong hyperlattice, if

(v) $a \in a \wedge b \Longrightarrow a \vee b=b$,

for all $a, b, c \in L$.

In a natural way, we can extend" $\wedge$ " and " $\vee "$ to subsets of $H$, as follows

$$
\begin{gathered}
A \wedge B=\cup\{a \wedge b \mid a \in A, b \in B\}, \\
A \vee B=\{a \vee b \mid a \in A, b \in B\},
\end{gathered}
$$

where, $A, B \in P^{*}(H)$.

Example 5. Let $(L, \vee, \wedge)$ be a lattice and define $a \oplus b=\{x \mid x \leq a \wedge b\}$. Then $(L, \vee, \oplus)$ is a $\wedge "$ - hyperlattice.

Remark 6. The converse of $(v)$ in Definition 4 is true. If $a \vee b=b$, by Definition 4 (iv), we have $a \in a \wedge(a \vee b)$. Since $a \vee b=b, a \in a \wedge b$. Then we define the relation $\leq$ on a strong $\wedge-$ hyperlattice $L$; it is clear that $(L, \leq)$ is a poset. $a \leq b \Longleftrightarrow a \in a \wedge b \Longleftrightarrow a \vee b=b$.

Definition 7. Let $L$ be $a \wedge$-hyperlattice. We say that $L$ is bounded if there exist $0,1 \in L$, such that $0 \leq x \leq 1$, for all $x \in L$. We say that 0 is the least element of $L$ and 1 is the greatest element of $L$.

Example 8. Let $L=\{0, a, 1\}$, and define $\wedge$-hyperoperation and $\vee$-operation on $L$ with tables 1. Then $(L, \wedge, \vee)$ is a bounded " $\wedge$ "-hyperlattice.

\begin{tabular}{c|ccc}
$\wedge$ & 0 & $a$ & 1 \\
\hline 0 & $\{0\}$ & $\{0\}$ & $\{0\}$ \\
$a$ & $\{0\}$ & $\{a, 0\}$ & $\{a, 0\}$ \\
1 & $\{0\}$ & $\{a, 0\}$ & $L$
\end{tabular}

(a)

\begin{tabular}{l|lll}
$\vee$ & 0 & $a$ & 1 \\
\hline 0 & 0 & $a$ & 1 \\
$a$ & $a$ & $a$ & 1 \\
1 & 1 & 1 & 1
\end{tabular}

(b)

Table 1 
M. Amiri Bideshki, R. Ameri, A. Borumand Saeid / Eur. J. Pure Appl. Math, 11 (1) (2018), 169-188 172

Proposition 9. Let $L$ be a bounded strong $\wedge$-hyperlattice. Then the following statements hold.

(i) If $a, b \neq 1$, and $a \vee b=1$, then $a, b \notin a \wedge b$;

(ii) If $a \wedge b=L$ or $a, b \in a \wedge b$, then $a=b$;

(iii) $\forall a \in L, a \in a \wedge 1$ and $0 \in x \wedge 0$.

Proof. (i) Let $a$ or $b \in a \wedge b$. If $a \in a \wedge b$, by Remark $6, a \vee b=b$, also we have $a \vee b=1$. So $b=1$, which is a contradiction. If $b \in a \wedge b$, similarly is proved that $a=1$ and it is a contradiction.

(ii): Let $a \wedge b=L$. Then $a, b \in a \wedge b$. So by Remark 6 and $a \in a \wedge b$, we have $a \vee b=b$. Also by $b \in a \wedge b$, we conclude $a \vee b=a$. So $a=b$.

(iii): $\forall a \in L$, we have $0 \leq a \leq 1$. So by Remark 6 , proof is obvious.

Definition 10. Let $L$ be $a \wedge-$ hyperlattice. Then:

(i) $L$ is called distributive if $a \vee(b \wedge c)=(a \vee b) \wedge(a \vee c)$, for all $a, b, c \in L$.

(ii) $L$ is called dual distributive if $a \wedge(b \vee c)=(a \wedge b) \vee(a \wedge c)$, for all $a, b, c \in L$.

(iii) $L$ is called strongly distributive if $L$ is both distributive and dual distributive.

Example 11. Let $L=\{a, b\}$. " $\wedge$ " hyperoperation and " $\vee "$ - operation are given with tables 2. Then $(L, \wedge, \vee)$ is a distributive " $\wedge$ "-hyperlattice, but since $b \wedge(a \vee a)=\{a\}$, and $(b \wedge a) \vee(b \wedge a)=\{a, b\}, L$ is not dual distributive.

\begin{tabular}{c|ccc|cc}
$\wedge$ & $a$ & $b$ & $\vee$ & $a$ & $b$ \\
\hline$a$ & $\{a, b\}$ & $\{a\}$ & $a$ & $a$ & $b$ \\
$b$ & $\{a\}$ & $\{b\}$ & $b$ & $b$ & $b$
\end{tabular}

(a)

Table 2:

Example 12. Let $L=\{a, b\}$. " $\wedge "$ - hyperoperation and $\vee "$ - operation are given with tables 3. Then $(L, \wedge, \vee)$ is a dual distributive $\wedge$-hyperlattice, but since $b \vee(a \wedge a) \neq$ $(b \vee a) \wedge(b \vee a), L$ is not distributive.

\begin{tabular}{c|cc}
$\wedge$ & $a$ & $b$ \\
\hline$a$ & $\{a\}$ & $\{a\}$ \\
$b$ & $\{a\}$ & $\{a, b\}$
\end{tabular}

(a)

\begin{tabular}{l|ll}
$\vee$ & $a$ & $b$ \\
\hline$a$ & $a$ & $b$ \\
$b$ & $b$ & $b$
\end{tabular}

(b)

Table 3 
M. Amiri Bideshki, R. Ameri, A. Borumand Saeid / Eur. J. Pure Appl. Math, 11 (1) (2018), 169-188 173

\begin{tabular}{c|cc}
$\wedge$ & $a$ & $b$ \\
\hline$a$ & $\{a, b\}$ & $\{a, b\}$ \\
$b$ & $\{a, b\}$ & $\{b\}$
\end{tabular}

(a)

\begin{tabular}{l|ll}
$\vee$ & $a$ & $b$ \\
\hline$a$ & $a$ & $b$ \\
$b$ & $b$ & $b$
\end{tabular}

(b)

Table 4

Example 13. Let $L=\{a, b\}$. " $\wedge$ "hyperoperation and $" \vee "$ operation are given with tables 4 , then $(L, \wedge, \vee)$ is a strongly distributive " $\wedge$ "-hyperlattice.

Proposition 14. If $L$ is distributive, then there is not $x \in a \wedge a$, such that $x \supsetneqq a$, for all $a \in L$.

Proof. Suppose that there exists $x \in a \wedge a$ such that $x \supsetneqq a$. So we have $a \vee x=a$, and since $L$ is distributive, we have $a \vee(a \wedge x)=(a \vee a) \wedge(a \vee x)=a \wedge a$. Since $x \in a \wedge a$, there exists $t \in a \wedge x$, such that $a \vee t=x$. we know that $a \leq a \vee t$ and it implies that $a \leq x$, which is a contradiction.

Lemma 15. For all $a, b \in L$, there exist $c, d \in a \wedge b$, such that $c \leq a$ and $d \leq b$.

Proof. Since $a \in a \vee(a \wedge b)$, then there exists $c \in a \wedge b$, such that $a=a \vee c$, and it implies that $c \leq a$. Also since $b \in b \vee(a \wedge b)$, there exists $d \in a \wedge b$, such that $b=b \vee d$, and implies $d \leq b$.

Theorem 16. Let $a, b, 1 \in L$. Then the following conditions hold.

(i) If $a \wedge b=\{1\}$, then $a=b$.

(ii) If $L$ is distributive, then $1 \wedge 1=\{1\}$.

Proof. ( $i$ ) By Proposition 15, $1 \leq a$ and $1 \leq b$, so they imply that $a=b=1$.

(ii) Since $L$ is distributive, $1 \vee(a \wedge b)=(1 \vee a) \wedge(1 \vee b)$. Also we have $1 \vee(a \wedge b)=\{1\}$ and $(1 \vee a) \wedge(1 \vee b)=1 \wedge 1$. So $1 \wedge 1=\{1\}$.

\section{Hyperideals and Hyperfilters in strong " $\wedge$ "-hyperlattices}

In this section, notaions of hyperideals (hyperfilters) in strong " $\wedge$ "-hyperlattices are given. In the sequel,$L$ denotes a strong " $\wedge$ "-hyperlattice.

Definition 17. Let $I$ and $F$ are nonempty subsets of $L$. Then:

(i) I is called a hyperideal if the following conditions hold.

(a) If $x, y \in I$, then $x \vee y \in I$,

(b) If $x \in I$ and $y \in L$, such that $y \leq x$, then $y \in I$.

(ii) F is called a hyperfilter if the following conditions hold. 
M. Amiri Bideshki, R. Ameri, A. Borumand Saeid / Eur. J. Pure Appl. Math, 11 (1) (2018), 169-188 174

(a) If $x, y \in F$, then $x \wedge y \subseteq F$,

(b) If $x \in F$ and $y \in L$, such that $x \leq y$, then $y \in F$.

(iii) A hyperideal $I$ is called prime if $(x \wedge y) \cap I \neq \emptyset$, then $x \in I$ or $y \in I$, for all $x, y \in L$.

(iv) A hyperfilter $F$ is called prime if $x \vee y \in F$, then $x \in F$ or $y \in F$, for all $x, y \in L$.

Example 18. (i) Let $L=\{0, a, b, 1\} . \wedge$-hyperoperation and $\vee$ operation are given with tables 5. Then $(L, \wedge, \vee)$ is a $\wedge$-hyperlattice. $\{0, a\}$ is an hyperideal, but since $b \wedge 1=$ $\{0, b\},(b \wedge 1) \cap\{0, a\} \neq \emptyset$, it is not a prime ideal. Also $\{0\}$ is an hyperideal that is not prime. $\{b, 1\}$ is neither a hyperideal nor a hyperfilter of $L$. Both $\{1\}$ and $L$ are hyperfilters of $L$ that $\{1\}$ is not prime.

\begin{tabular}{c|cccc}
$\wedge$ & 0 & $a$ & $b$ & 1 \\
\hline 0 & $\{0\}$ & $\{0\}$ & $\{0\}$ & $\{0\}$ \\
$a$ & $\{0\}$ & $\{0, a\}$ & $\{0\}$ & $\{0, a\}$ \\
$b$ & $\{0\}$ & $\{0\}$ & $\{0, b\}$ & $\{0, b\}$ \\
1 & $\{0\}$ & $\{a\}$ & $\{b\}$ & $\{1\}$
\end{tabular}

(a)

\begin{tabular}{l|llll}
$\vee$ & 0 & $a$ & $b$ & 1 \\
\hline 0 & 0 & $a$ & $b$ & 1 \\
$a$ & $a$ & $a$ & 1 & 1 \\
$b$ & $b$ & 1 & $b$ & 1 \\
1 & 1 & 1 & 1 & 1
\end{tabular}

(b)

Table 5:

(ii) Consider" $\wedge "$-hyperlattice $L$ in Example 8. Then $\{0, a\}$ is a prime hyperideal.

Proposition 19. Let $F$ be a nonempty subset of $L$. Then $F$ is a hyperfilter if and only if $F$ is " $\wedge "$-closed and for all $x \in L$ and $a \in F, x \vee a \in F$.

Let $L$ be a bounded " $\wedge$ "-hyperlattice. It is clear that if $I$ is a hyperideal of $L$ and $1 \in I$, then $I=L$. Also if $F$ is a hyperfilter of $L$ and $0 \in F$, then $F=L$. If $1 \wedge 1=L$, then hyperlattice $L$ does not have trivial hyperfilter. We have $0 \in I$, and $1 \in F$, where $I$ is a hyperideal and $F$ is a hyperfilter of $L$.

Now, we are going to define two types of hyperideals in a " $\wedge$ "-hyperlattice $L$.

Proposition 20. Let $L$ be dual distributive. If $\forall i \in I, L_{i}$ is a hyperideal of $L$, then $\cap_{i \in I} L_{i}$ is a hyperideal of $L$

Proposition 21. Let $I, J$ are hyperideals of $L$. Then $I \cup J$ is hyperideal if and only if $I \subseteq J$ or $J \subseteq I$.

Theorem 22. Let $I, J$ be hyperideals of $L$. Define $I \vee J=\{x \in L \mid x \leq a \vee b, \exists a \in I, b \in$ $J\}$. Then $I \vee J$ is a hyperideal of $L$ and we have:

$$
I \vee J=\{x \in L \mid \exists a \in I, \exists b \in J, x \in x \wedge(a \vee b)\} .
$$

Theorem 23. Let $L$ be dual distributive that contains the least element 0 and $a \in L$. Then: 
(i) $I_{a}=\{x \in L \mid 0 \in x \wedge a\}$ is a hyperideal of $L$.

(ii) $I(a)=\{x \in L \mid x \in x \wedge a\}$ is the least hyperideal of $L$ that contains a.

Proof. (i): Let $x, y \in I_{a}$. So $0 \in x \wedge a$ and $0 \in y \wedge a$. We have $0 \vee 0=0$, therefore $0 \in(x \wedge a) \vee(y \wedge a)$, and since $L$ is dual distributive, $0 \in a \wedge(x \vee y)$, it implies that $x \vee y \in I_{a}$. now, Let $x \in I_{a}$, and $y \leq x$. So $0 \in x \wedge a$ and $x \vee y=x$. Thus we have $0 \in(x \vee y) \wedge a$, and since $l$ is dual distributive, $0 \in(x \wedge a) \vee(y \wedge a)$. Since $0 \in(x \wedge a)$ and $0 \vee 0=0$, we conclude $0 \in y \wedge a$. (ii): Let $x, y \in I(a)$. Then $a \in x \wedge a$ and $a \in y \wedge a$. Since $L$ is dual distributive and $a \vee a=a$, we have $a \in(x \wedge a) \vee(y \wedge a)=a \wedge(x \vee y)$ and it implies that $x \vee y \in I(a)$. Now, Let $x \in I(a)$, and $y \leq x$. So $x \in x \wedge a$, and $y \in x \wedge y$. Thus we have $a \vee x=a$ and $x \vee y=x$, so $a \vee y=(a \vee x) \vee y=a \vee(x \vee y)=a \vee x=a$. Then $a \vee y=a$ and it implies that $y \in a \wedge y$; Therefore $y \in I(a)$.

Corollary 24. Let $L$ be bounded dual distributive. Then we have:

(i) $I(0)=\{0\}$;

(ii) $I(1)=L$;

(iii) If $a, b \in L$, and $a \leq b$, then $I(a) \subseteq I(b)$, and $I_{b} \subseteq I_{a}$;

(iv) $I_{0}=L$;

(v) $I(a), I(b) \subseteq I(a \vee b)$.

Lemma 25. Let $L$ be dual distributive. If $I$ is a hyperideal of $L$ and $a \in L$, then $I \vee I(a)$ is a " $\vee "$-closed subset of $L$.

Proof. Let $x, y \in I \vee I(a)$. So there exist $p_{1}, p_{2} \in I$ and $a_{1}, a_{2} \in I(a)$, such that $x=p_{1} \vee a_{1}, y=p_{2} \vee a_{2}$. We have:

$$
x \vee y=p_{1} \vee a_{1} \vee p_{2} \vee a_{2}=\left(p_{1} \vee p_{2}\right) \vee\left(a_{1} \vee a_{2}\right) .
$$

We know $p_{1} \vee p_{2} \in I$ and $a_{1} \vee a_{2} \in I(a)$. Therefore $x \vee y \in I \vee I(a)$.

Definition 26. Let $L$ be distributive and $A \subseteq L$. Then the least hyperfilter of $L$ that contains $A$, is called the generating hyperfilter by subset $A$, and it is denoted by $F(A)$.

Proposition 27. Let $L$ be distributive and $a \in L$. Then $F(a)=\{x \in L \mid a \in a \wedge x\}=$ $\{x \in L \mid a \leq x\}$.

Corollary 28. Let $L$ be a bounded distributive " $\wedge$ "-hyperlattice and $a, b \in L$. Then the following conditions hold.

(i) $F(1)=\{1\}$. 
M. Amiri Bideshki, R. Ameri, A. Borumand Saeid / Eur. J. Pure Appl. Math, 11 (1) (2018), 169-188 176

(ii) $F(0)=L$.

(iii) $F(a) \vee F(b)=F(a \vee b)$.

(iv) If $a \leq b$, then $F(b) \subseteq F(a)$.

Lemma 29. Let $L$ be a distributive " $\wedge$ "-hyperlattice and $a, 1 \in L$, such that $a \notin F$. If $F$ is a hyperfilter of $L$, then $F \wedge F(a)$ is so. Also $F, F(a) \varsubsetneqq(F \wedge F(a))$.

Proof. We prove $F, F(a) \varsubsetneqq F \wedge F(a)$, only. Let $x \in F$. We have $x \leq 1$ so, by Remark $6, x \in x \wedge 1$, and since $1 \in F(a)$, then $x \in F \wedge F(a)$. Now, suppose that $x \in F(a)$. So $x \in 1 \wedge x$, and it implies that $x \in F \wedge F(a)$.

Theorem 30. Let $L$ be a distributive strong $\wedge-$ hyperlattice and $a, b \in L$. If $P$ is $a$ hyperfilter of $L$, then $(P \wedge F(a)) \vee(P \wedge F(b))=P \wedge F(a \vee b)$.

Proof. Let $x \in P \wedge(F(a \vee b)$. So there exist $p \in P$, and $c \in F(a \vee b)$, such that $x \in p \wedge c$, and $a \vee b \leq c$. We have $a \leq a \vee b, a \vee b \leq c$, and $\leq$ is transitive, so $a \leq c$ and it implies that $c \in F(a)$. Therefore $x \in P \wedge F(a)$. Similarly, it is proved that $x \in P \wedge F(b)$. Since $x \vee x=x, x \in(P \wedge F(a)) \vee(P \wedge F(b))$, it implies that:

$$
P \wedge F(a \vee b) \subseteq(P \wedge F(a)) \vee(P \wedge F(b)) .
$$

Let $x \in(P \wedge F(a)) \vee(P \wedge F(b))$. So there exist $p_{1}, p_{2} \in P, a_{1} \in F(a), b_{1} \in F(b)$, such that $x \in\left(p_{1} \wedge a_{1}\right) \vee\left(p_{2} \wedge b_{1}\right)$, and $a \leq a_{1}, b \leq b_{1}$. Since $L$ is distributive, we have:

$$
\begin{aligned}
x \in\left(p_{1} \wedge a_{1}\right) \vee\left(p_{2} \wedge b_{1}\right)= & {\left[\left(p_{1} \wedge a_{1}\right) \vee p_{2}\right] \wedge\left[\left(p_{1} \wedge a_{1}\right) \vee b_{1}\right] } \\
= & \left(p_{2} \vee p_{1}\right) \wedge\left(p_{2} \vee a_{1}\right) \wedge\left(b_{1} \vee p_{1}\right) \wedge\left(b_{1} \vee a_{1}\right) .
\end{aligned}
$$

Since $P$ is a hyperfilter, $p_{2} \leq p_{2} \vee p_{1}, p_{2} \leq p_{2} \vee a_{1}$, and $p_{1} \leq b_{1} \vee p_{1}$, then $p_{2} \vee p_{1}, p_{2} \vee$ $a_{1}, b_{1} \vee p_{1} \in P$. So, we conclude that

$$
\left(p_{2} \vee p_{1}\right) \wedge\left(p_{2} \vee a_{1}\right) \wedge\left(b_{1} \vee p_{1}\right) \subseteq P
$$

Since $a \leq a_{1}, b \leq b_{1}, a \vee b \leq a_{1} \vee b_{1}$, and it implies that $a_{1} \vee b_{1} \in F(a \vee b)$. So $x \in P \wedge F(a \vee b)$. Thus:

$$
(P \wedge F(a)) \vee(P \wedge F(b)) \subseteq P \wedge F(a \vee b)
$$

Theorem 31. Let $x, y \in L$. If $x \wedge y$ is a hyperfilter of $L$, then $x=y$.

Proof. Let $x \wedge y$ be a hyperfilter. By Lemma 15, there exist $a, b \in x \wedge y$, such that $a \leq x$ and $b \leq y$. Then $x, y \in x \wedge y$ and by Proposition $9,(i i), x=y$. 


\section{4. $I_{A^{-}}$Hyperideals in strong $" \wedge "$-hyperlattices}

In this section, we are going to define some types of hyperideals in a dual distributive $" \wedge "$-hyperlattices. We assume that $L$ is a dual distributive and bounded strong " $\wedge "$ hyperlattice.

Theorem 32. Let $\emptyset \neq A \subseteq L$. If

$$
I_{A}=\{x \in L \mid 0 \in x \wedge a, \forall a \in A\},
$$

then $I_{A}$ is a hyperideal of $L$.

Proof. Since $0 \leq a, \forall a \in A$, by Remark $6,0 \in 0 \wedge a$, and it implies that $0 \in I_{A}$, and $I_{A} \neq \emptyset$. Let $x, y \in I_{A}$. Then $0 \in x \wedge a$, and $0 \in y \wedge a$, for all $a \in A$. So $0 \in(x \wedge a) \vee(y \wedge a)$, for all $a \in A$; since $L$ is dual distributive, $0 \in(x \vee y) \wedge a$, for all $a \in A$. So $x \vee y \in I_{A}$. Now, Let $x \in I_{A}$ and $y \leq x$. Then $0 \in x \wedge a$, for all $a \in A$, and $x \vee y=x$. We have $0 \in x \wedge a=(x \vee y) \wedge a$, for all $a \in A$, and since $L$ is dual distributive, $0 \in(x \wedge a) \vee(y \wedge a)$, for all $a \in A$. Since $0 \vee 0=0$ and $0 \in x \wedge a$, for all $a \in A$, we conclude that $0 \in y \wedge a$, for all $a \in A$, and it implies that $y \in I_{A}$. So $I_{A}$ is a hyperideal of $L$.

Corollary 33. Let $L$ be $a \wedge$-hyperlattice and $A, B \subseteq L$. Then we have:

(i) $I_{A} \subseteq I_{a}, \forall a \in A$;

(ii) If $A \subseteq B$, then $I_{B} \subseteq I_{A}$;

(iii) $I_{A}=\cap\left\{I_{a}: a \in A\right\}$.

(iv) $I_{A} \cap I_{B}=I_{A \cup B}$.

Now, we define the least hyperideal generating by $A \subseteq L$, and we denote it by $I(A)$.

Definition 34. Let $A \subseteq L$. We define $I(A)=\bigcap_{A \subseteq I}\{I \mid I$ is a hyperideal of $L\}$, and $I(A)$ is said to be the least hyperideal generating by $\bar{A}$.

Example 35. (i) Consider $\wedge-$ hyperlattice $L$ in Example 18. Let $I(L)$ be the set of all hyperideals of $L$. Then we have $I(L)=\{\{0\},\{0, a\},\{0, b\}, L\}$. If $A=\{a, b\}$, then $I(A)=L$. Also, if $A=\{a\}$, then $I(a)=\{0, a\}$.

(ii) Consider $\wedge-$ hyperlattice $L$ in Example 13. Let $A=\{a\}$ and $B=\{b\}$. Then $I_{A}=L$ and $I_{B}=\{a\}$. Also, we have $B \cap I_{B}=\emptyset$.

Proposition 36. The following statements hold.

(i) $I(\emptyset)=\{0\}$;

(ii) $I(\{0\})=\{0\}$; 
M. Amiri Bideshki, R. Ameri, A. Borumand Saeid / Eur. J. Pure Appl. Math, 11 (1) (2018), 169-188 178

(iii) If $A \subseteq B$, then $I(A) \subseteq I(B)$.

Proposition 37. If $a, b \in L$, such that $a \leq b$, then $I(a) \subseteq I(a \wedge b)$.

Proof. Let $a, b \in L$, such that $a \leq b$. By Remark $6, a \in a \wedge b$, and it implies that $\{a\} \subseteq(a \wedge b)$. So by Proposition 48, (iii), $I(a) \subseteq I(a \wedge b)$.

Theorem 38. $I(A)=\left\{x \in L \mid x \in x \wedge\left(a_{1} \vee a_{2} \vee \ldots \vee a_{n}\right), \exists a_{1}, \ldots, a_{n} \in A\right\}$

Proof. Let $B=\left\{x \in L \mid x \in x \wedge\left(a_{1} \vee a_{2} \vee \ldots \vee a_{n}\right), \exists a_{1}, \ldots, a_{n} \in A\right\}$. We show that $B$ is a hyperideal and we show that if $I$ is a hyperideal, such that $A \subseteq I$, then $B \subseteq I$.

Assume $x, y \in B$. So there exist $a_{1}, a_{2}, \ldots, a_{n}, b_{1}, b_{1}, b_{2}, \ldots, b_{m} \in A$, such that:

$$
\begin{aligned}
& x \in x \wedge\left(a_{1} \vee a_{2} \vee \ldots \vee a_{n}\right), \\
& y \in y \wedge\left(b_{1} \vee b_{2} \vee \ldots \vee b_{m}\right) .
\end{aligned}
$$

So, we have:

$x \vee\left(a_{1} \vee a_{2} \vee \ldots \vee a_{n}\right)=\left(a_{1} \vee a_{2} \vee \ldots \vee a_{n}\right), y \vee\left(b_{1} \vee b_{2} \vee \ldots \vee b_{m}\right)=\left(b_{1} \vee b_{2} \vee \ldots \vee b_{m}\right)$. Also, we have:

$$
\begin{aligned}
& x \vee y \vee\left(a_{1} \vee a_{2} \vee \ldots \vee a_{n} \vee b_{1} \vee b_{2} \vee \ldots \vee b_{m}\right)= \\
& =\left[x \vee\left(a_{1} \vee a_{2} \vee \ldots \vee a_{n}\right)\right] \vee\left[y \vee\left(b_{1} \vee b_{2} \vee \ldots \vee b_{m}\right)\right] \\
& =\left(a_{1} \vee a_{2} \vee \ldots \vee a_{n}\right) \vee\left(b_{1} \vee b_{2} \vee \ldots \vee b_{m}\right) .
\end{aligned}
$$

So $x \vee y \in(x \vee y) \wedge\left(a_{1} \vee a_{2} \vee \ldots \vee a_{n} \vee b_{1} \vee b_{2} \vee \ldots \vee b_{m}\right)$.

Let $x \in B$, and $y \leq x$. Therefore there exist $a_{1}, a_{2}, \ldots, a_{n} \in A$, such that $x \in x \wedge\left(a_{1} \vee a_{2} \vee\right.$ $\ldots \vee a_{n}$ ), By Remark 6, we have $x \leq a_{1} \vee a_{2} \vee \ldots \vee a_{n}$, since $y \leq x$, and $\leq$ is a transitive relation, $y \leq\left(a_{1} \vee a_{2} \vee \ldots \vee a_{n}\right)$, it implies that $y \in y \wedge\left(a_{1} \vee a_{2} \vee \ldots \vee a_{n}\right)$. So $y \in B$. We showed that $B$ is a hyperideal. Now, we show that if $I$ is a hyperideal of $L$, such that $A \subseteq I$, then $B \subseteq I$. Let $x \in B$. Therefore there exist $a_{1}, a_{2}, \ldots, a_{n} \in A$, such that $x \in x \wedge\left(a_{1} \vee a_{2} \vee \ldots \vee a_{n}\right)$, and it implies that $x \leq a_{1} \vee a_{2} \vee \ldots \vee a_{n}$. Since $A \subseteq I$, and $I$ is a hyperideal, $a_{1} \vee a_{2} \vee \ldots \vee a_{n} \in I$. We have $x \leq a_{1} \vee a_{2} \vee \ldots \vee a_{n} \in I$, then $x \in I$. So $B \subseteq I$.

Definition 39. Let $\emptyset \neq S \subseteq L$. Then $S$ is called a subhyperlattice if $x \vee y \in S$, and $x \wedge y \subseteq S$, for all $x, y \in S$.

Example 40. Consider $\wedge$-hyperlattice $L$ in Example 18. It is clear that $\{0, a, 1\}$ is a $\wedge$-subhyperlattice of $L$, but $\{b, 1\}$ is not a $\wedge$-subhyperlattice, since $b \wedge b=\{0, b\}$ and $\{0, b\} \nsubseteq\{b, 1\}$. Also, both $\{0\}$ and $\{1\}$ are " $\wedge "$-subhyperlattices of $L$.

Theorem 41. Let $I \subseteq L$. If the following conditions hold, then $I$ is a hyperideal of $L$.

(i) I is " $\vee "$-closed,

(ii) If $a \in I$, and $x \in L$, then $a \wedge x \subseteq I$, 
Proof. It is enough prove that If $a \in I$, and $x \leq a$, then $x \in I$. By $(i i), a \wedge x \subseteq I$. It is clear that $x \in x \wedge a$, and since $a \wedge x \subseteq I, x \in I$.

Remark 42. The converse of Theorem 53 does not hold. Consider the " $\wedge "$ - hyperlattice $L$ in Example 11. It is clear that $\{a\}$ is a hyperideal of $L$; Since $a \wedge a=\{a, b\}$, condition (ii) in Theorem 53 does not hold. So, we conclude that concept of hyperideal in hyperlattice and concept of ideal in lattice are different.

If $I \subseteq L$ is both a subhyperlattice and a hyperideal, then condition (ii) in Theorem 53 , is satisfied. So we state the following theorem.

Theorem 43. A subhyperlattice $I$ is a hyperideal if and only if $a \wedge x \subseteq I$, where $a \in I$, and $x \in L$.

\section{5. $I_{A^{-}}$Hyperideals in strong $" \wedge "$-hyperlattices}

In this section, we are going to define some types of hyperideals in a dual distributive $" \wedge$ "-hyperlattices. We assume that $L$ is a dual distributive and bounded strong " $\wedge "$ hyperlattice.

Theorem 44. Let $\emptyset \neq A \subseteq L$. If

$$
I_{A}=\{x \in L \mid 0 \in x \wedge a, \forall a \in A\},
$$

then $I_{A}$ is a hyperideal of $L$.

Proof. Since $0 \leq a, \forall a \in A$, by Remark $6,0 \in 0 \wedge a$, and it implies that $0 \in I_{A}$, and $I_{A} \neq \emptyset$. Let $x, y \in I_{A}$. Then $0 \in x \wedge a$, and $0 \in y \wedge a$, for all $a \in A$. So $0 \in(x \wedge a) \vee(y \wedge a)$, for all $a \in A$; since $L$ is dual distributive, $0 \in(x \vee y) \wedge a$, for all $a \in A$. So $x \vee y \in I_{A}$. Now, Let $x \in I_{A}$ and $y \leq x$. Then $0 \in x \wedge a$, for all $a \in A$, and $x \vee y=x$. We have $0 \in x \wedge a=(x \vee y) \wedge a$, for all $a \in A$, and since $L$ is dual distributive, $0 \in(x \wedge a) \vee(y \wedge a)$, for all $a \in A$. Since $0 \vee 0=0$ and $0 \in x \wedge a$, for all $a \in A$, we conclude that $0 \in y \wedge a$, for all $a \in A$, and it implies that $y \in I_{A}$. So $I_{A}$ is a hyperideal of $L$.

Corollary 45. Let $L$ be $a \wedge$-hyperlattice and $A, B \subseteq L$. Then we have:

(i) $I_{A} \subseteq I_{a}, \forall a \in A$;

(ii) If $A \subseteq B$, then $I_{B} \subseteq I_{A}$;

(iii) $I_{A}=\cap\left\{I_{a}: a \in A\right\}$.

(iv) $I_{A} \cap I_{B}=I_{A \cup B}$.

Now, we define the least hyperideal generating by $A \subseteq L$, and we denote it by $I(A)$. 
M. Amiri Bideshki, R. Ameri, A. Borumand Saeid / Eur. J. Pure Appl. Math, 11 (1) (2018), 169-188 180

Definition 46. Let $A \subseteq L$. We define $I(A)=\bigcap_{A \subseteq I}\{I \mid I$ is a hyperideal of $L\}$, and $I(A)$ is said to be the least hyperideal generating by $\bar{A}$.

Example 47. (i) Consider $\wedge-$ hyperlattice $L$ in Example 18. Let $I(L)$ be the set of all hyperideals of $L$. Then we have $I(L)=\{\{0\},\{0, a\},\{0, b\}, L\}$. If $A=\{a, b\}$, then $I(A)=L$. Also, if $A=\{a\}$, then $I(a)=\{0, a\}$.

(ii) Consider $\wedge$ - hyperlattice $L$ in Example 13. Let $A=\{a\}$ and $B=\{b\}$. Then $I_{A}=L$ and $I_{B}=\{a\}$. Also, we have $B \cap I_{B}=\emptyset$.

Proposition 48. The following statements hold.

(i) $I(\emptyset)=\{0\}$;

(ii) $I(\{0\})=\{0\}$;

(iii) If $A \subseteq B$, then $I(A) \subseteq I(B)$.

Proposition 49. If $a, b \in L$, such that $a \leq b$, then $I(a) \subseteq I(a \wedge b)$.

Proof. Let $a, b \in L$, such that $a \leq b$. By Remark $6, a \in a \wedge b$, and it implies that $\{a\} \subseteq(a \wedge b)$. So by Proposition 48, (iii), $I(a) \subseteq I(a \wedge b)$.

Theorem 50. $I(A)=\left\{x \in L \mid x \in x \wedge\left(a_{1} \vee a_{2} \vee \ldots \vee a_{n}\right), \exists a_{1}, \ldots, a_{n} \in A\right\}$

Proof. Let $B=\left\{x \in L \mid x \in x \wedge\left(a_{1} \vee a_{2} \vee \ldots \vee a_{n}\right), \exists a_{1}, \ldots, a_{n} \in A\right\}$. We show that $B$ is a hyperideal and we show that if $I$ is a hyperideal, such that $A \subseteq I$, then $B \subseteq I$.

Assume $x, y \in B$. So there exist $a_{1}, a_{2}, \ldots, a_{n}, b_{1}, b_{1}, b_{2}, \ldots, b_{m} \in A$, such that:

$$
\begin{aligned}
& x \in x \wedge\left(a_{1} \vee a_{2} \vee \ldots \vee a_{n}\right), \\
& y \in y \wedge\left(b_{1} \vee b_{2} \vee \ldots \vee b_{m}\right) .
\end{aligned}
$$

So, we have:

$$
\begin{aligned}
& x \vee\left(a_{1} \vee a_{2} \vee \ldots \vee a_{n}\right)=\left(a_{1} \vee a_{2} \vee \ldots \vee a_{n}\right), \\
& y \vee\left(b_{1} \vee b_{2} \vee \ldots \vee b_{m}\right)=\left(b_{1} \vee b_{2} \vee \ldots \vee b_{m}\right) .
\end{aligned}
$$

Also, we have:

$$
\begin{aligned}
& x \vee y \vee\left(a_{1} \vee a_{2} \vee \ldots \vee a_{n} \vee b_{1} \vee b_{2} \vee \ldots \vee b_{m}\right)= \\
& =\left[x \vee\left(a_{1} \vee a_{2} \vee \ldots \vee a_{n}\right)\right] \vee\left[y \vee\left(b_{1} \vee b_{2} \vee \ldots \vee b_{m}\right)\right] \\
& =\left(a_{1} \vee a_{2} \vee \ldots \vee a_{n}\right) \vee\left(b_{1} \vee b_{2} \vee \ldots \vee b_{m}\right) .
\end{aligned}
$$

So $x \vee y \in(x \vee y) \wedge\left(a_{1} \vee a_{2} \vee \ldots \vee a_{n} \vee b_{1} \vee b_{2} \vee \ldots \vee b_{m}\right)$.

Let $x \in B$, and $y \leq x$. Therefore there exist $a_{1}, a_{2}, \ldots, a_{n} \in A$, such that $x \in x \wedge\left(a_{1} \vee a_{2} \vee\right.$ 
$\ldots \vee a_{n}$ ), By Remark 6 , we have $x \leq a_{1} \vee a_{2} \vee \ldots \vee a_{n}$, since $y \leq x$, and $\leq$ is a transitive relation, $y \leq\left(a_{1} \vee a_{2} \vee \ldots \vee a_{n}\right)$, it implies that $y \in y \wedge\left(a_{1} \vee a_{2} \vee \ldots \vee a_{n}\right)$. So $y \in B$. We showed that $B$ is a hyperideal. Now, we show that if $I$ is a hyperideal of $L$, such that $A \subseteq I$, then $B \subseteq I$. Let $x \in B$. Therefore there exist $a_{1}, a_{2}, \ldots, a_{n} \in A$, such that $x \in x \wedge\left(a_{1} \vee a_{2} \vee \ldots \vee a_{n}\right)$, and it implies that $x \leq a_{1} \vee a_{2} \vee \ldots \vee a_{n}$. Since $A \subseteq I$, and $I$ is a hyperideal, $a_{1} \vee a_{2} \vee \ldots \vee a_{n} \in I$. We have $x \leq a_{1} \vee a_{2} \vee \ldots \vee a_{n} \in I$, then $x \in I$. So $B \subseteq I$.

Definition 51. Let $\emptyset \neq S \subseteq L$. Then $S$ is called a subhyperlattice if $x \vee y \in S$, and $x \wedge y \subseteq S$, for all $x, y \in S$.

Example 52. Consider $\wedge$-hyperlattice $L$ in Example 18. It is clear that $\{0, a, 1\}$ is a $\wedge$-subhyperlattice of $L$, but $\{b, 1\}$ is not $a \wedge$-subhyperlattice, since $b \wedge b=\{0, b\}$ and $\{0, b\} \nsubseteq\{\{b, 1\}$. Also, both $\{0\}$ and $\{1\}$ are $" \wedge "$-subhyperlattices of $L$.

Theorem 53. Let $I \subseteq L$. If the following conditions hold, then $I$ is a hyperideal of $L$.

(i) $I$ is " $\vee "$-closed,

(ii) If $a \in I$, and $x \in L$, then $a \wedge x \subseteq I$,

Proof. It is enough prove that If $a \in I$, and $x \leq a$, then $x \in I$. By (ii), $a \wedge x \subseteq I$. It is clear that $x \in x \wedge a$, and since $a \wedge x \subseteq I, x \in I$.

Remark 54. The converse of Theorem 53 does not hold. Consider the " $\wedge$ "- hyperlattice $L$ in Example 11. It is clear that $\{a\}$ is a hyperideal of $L$; Since $a \wedge a=\{a, b\}$, condition (ii) in Theorem 53 does not hold. So, we conclude that concept of hyperideal in hyperlattice and concept of ideal in lattice are different.

If $I \subseteq L$ is both a subhyperlattice and a hyperideal, then condition (ii) in Theorem 53 , is satisfied. So we state the following theorem.

Theorem 55. A subhyperlattice $I$ is a hyperideal if and only if $a \wedge x \subseteq I$, where $a \in I$, and $x \in L$.

\section{Some Results in Prime Hyperideals of Strong " $\wedge$ "Hyperlattices}

Proposition 56. Let $P \subseteq L$, and $P$ is $\vee$-closed. Then $P$ is a prime hyperideal if and only if the following statements hold.

(i) If $a, b \notin P$, then $a \wedge b \subseteq L \backslash P$,

(ii) If $a \in P$, and $a \in a \wedge x$, then $x \in P$.

Theorem 57. If $P$ is a prime hyperideal of $L$, then $L \backslash P$ is a hyperfilter of $L$.

Proof. Let $x, y \in L \backslash P$. Then $x, y \notin P$. Since $P$ is a prime hyperideal, $(x \wedge y) \cap P=\emptyset$, so $(x \wedge y) \subseteq L \backslash P$. Let $a \in L \backslash P$, and $x \in L$, such that $a \leq x$. So we have $a \notin P$, and since $P$ is a hyperideal, $x \notin P$, it implies that $x \in L \backslash P$.

In Theorem 57, $P$ must be prime and the converse of it is not true. 
M. Amiri Bideshki, R. Ameri, A. Borumand Saeid / Eur. J. Pure Appl. Math, 11 (1) (2018), 169-188 182

Example 58. Consider" $\wedge$ "-hyperlattice $L$ in Example 18. Then $\{0\}$ is a hyperideal of L. $L \backslash\{0\}=\{a, b, 1\}$. Since $a \wedge b=\{0\}$ and $0 \notin\{a, b, 1\},\{a, b, 1\}$ is not a hyperfilter.

$\{b, 1\}$ is a hyperfilter, but $L \backslash\{0, a\}$ is not a prime hyperideal. We have $(b \wedge b) \cap\{0, a\} \neq \emptyset$, but $b \notin\{0, a\}$.

Theorem 59. $P$ is a prime hyperideal of $L$ if and only if $L \backslash P$ is a prime hyperfilter of $L$.

Proof. Let $P$ be a prime hyperideal of $L$. By Theorem 57, $L \backslash P$ is a hyperfilter of $L$. Now, assume $x, y \in L, x \vee y \in L \backslash P$, but $x, y \notin L \backslash P$. So, we have $x \vee y \notin P$, but $x, y \in P$; Since $P$ is a hyperideal, $x \vee y \in P$, which is a contradiction.

Conversely, let $L \backslash P$ is a prime hyperfilter of $L$. We assume $x, y \in P$. So $x, y \notin L \backslash P$, since $L \backslash P$ is a prime hyperfilter of $L, x \vee y \notin L \backslash P$, and it implies that $x \vee y \in P$.

Assume $a \in P, x \in L$, and $x \leq a$. If $x \notin P$, then $x \in L \backslash P$, and since $L \backslash P$ is a hyperfilter, $a \in L \backslash P$, which is a contradiction. Therefore $P$ is a hyperideal of $L$. Let $(x \wedge y) \cap P \neq \emptyset$, but $x, y \notin P$. So $x, y \in L \backslash P$; Since $L \backslash P$ is a hyperfilter, $x \wedge y \subseteq L \backslash P$, and it implies that $(x \wedge y) \cap P=\emptyset$, which is a contradiction. Thus $P$ is a prime hyperfilter.

Theorem 60. Let $L$ be dual distributive, $A, B \subseteq L$, and $P$ is a prime hyperideal of $L$. Then the following conditions hold.

(i) If $A \wedge B \subseteq P$, then $A \subseteq P$ or $B \subseteq P$.

(ii) If $I(a) \wedge I(b) \subseteq P$, then $a \in P$ or $b \in P$.

Proof. (i): Let $A \nsubseteq P$ and $B \nsubseteq P$. So there exist $a \in A \backslash P$ and $b \in B \backslash P$; We have $a \wedge b \subseteq A \wedge B \subseteq P$, so $a \wedge b \subseteq P$, and since $P$ is a prime hyperideal, $a \in P$ or $b \in P$, which is a contradiction.

(ii): Let $I(a) \wedge I(b) \subseteq P$. By $(i), I(a) \subseteq P$ or $I(b) \subseteq P$. Since $a \in I(a)$ and $b \in I(b)$, we conclude that $a \in P$ or $b \in P$.

Theorem 61. Let $L$ be distributive, $A, B \subseteq L$, and $D$ is a hyperfilter of $L$. Then the following conditions are equivalent.

(i) $D$ is a prime hyperfilter.

(ii) $L \backslash D$ is a prime hyperideal.

(iii) If $A \vee B \subseteq D$, then $A \subseteq D$ or $B \subseteq D$.

(iv) If $F(a) \vee F(b) \subseteq D$, then $a \in D$ or $b \in D$, for all $a, b \in L$.

Proof. By Theorem 59, (i), (ii) are equivalent.

(i) $\Longrightarrow($ iii): Let $A \vee B \subseteq D$, and $A \nsubseteq D, B \nsubseteq D$. So there exist $a \in A \backslash D$, and $b \in B \backslash D$, since $a \vee b \in A \vee B$, and $A \vee B \subseteq D, a \vee b \in D$; Since $D$ is a prime hyperfilter, $a \in D$ and $b \in D$, which is a contradiction. 
$($ iii $) \Longrightarrow($ iv): It is obvious.

$(i v) \Longrightarrow(1)$ : Let $x, y \in L$, and $(x \vee y) \in D$. Assume that $x \notin D$, and $y \notin D$. By (iv), $F(x) \vee F(y) \nsubseteq D$. Therefore there exists $c \in(F(x) \vee F(y)) \backslash D$. So there exist $a \in F(x), b \in F(y)$, such that $c=a \vee b$ and $x \leq a, y \leq b$. So, we have $a \vee b \notin D$, and $x \vee y \leq a \vee b$. Since $D$ is a hyperfilter, $x \vee y \notin D$, which is a contradiction.

Theorem 62 (Prime Hyperfilter Theorem). Let $L$ be distributive. If I is a hyperideal and $F$ is a hyperfilter of $L$, such that $I \cap F=\emptyset$, then there exists prime hyperfilter $D$ of $L$, such that $F \subseteq D$ and $I \cap D=\emptyset$.

Proof. Let $\Sigma=\{J \subseteq L \mid J$ is a hyperfilter and $J \cap I=\emptyset\}$. Since $F \in \Sigma, \Sigma \neq \emptyset$. Suppose $\left\{L_{i}\right\}_{i \in I}$ is a chain in $\Sigma$. We show that $\bigcup_{i \in I} L_{i} \in \Sigma$. Let $a, b \in \bigcup_{i \in I} L_{i}$. So there exist $i, j \in I$, such that $a \in L_{i}$, and $b \in L_{j}$; Since $\left\{L_{i}\right\}_{i \in I}$ is a chain, $L_{i} \subseteq L_{j}$ or $L_{j} \subseteq L_{i}$. We assume $L_{i} \subseteq L_{j}$. So $a, b \in L_{j}$ and since $L_{j}$ is a hyperfilter, $a \wedge b \in L_{j}$. Thus $a \wedge b \in \bigcup_{i \in I} L_{i}$. Let $a \in \bigcup_{i \in I} L_{i}$ and $x \geq a$. So there exists $i \in I$, such that $a \in L_{i}$. Since $L_{i}$ is a hyperfilter and $x \geq a, x \in L_{i}$, it implies that $x \in \bigcup_{i \in I} L_{i}$. So $\bigcup_{i \in I} L_{i}$ is a hyperfilter of $L$. We claim that $\left(\bigcup_{i \in I} L_{i}\right) \cap I=\emptyset$. If $\left(\bigcup_{i \in I} L_{i}\right) \cap I \neq \emptyset$, then there exists $a \in \bigcup_{i \in I} L_{i}$, and $a \in I$. So there exists $i \in I$, such that $a \in L_{i}$ and $a \in I$, it implies that $L_{i} \cap I \neq \emptyset$, which is a contradiction. Thus $\bigcup_{i \in I} L_{i} \in \Sigma$. By Zorn lemma, $\Sigma$ has a maximal element $D$. We claim that $D$ is a Prime hyperfilter. Let $a, b \in L$, and $(a \vee b) \in D$. We suppose that $a \notin D$ and $b \notin D$. Set $F_{1}=D \wedge F(a)$ and $F_{2}=D \wedge F(b)$. Since $a \in F_{1}, D \varsubsetneqq F_{1}$; Also we have $D \varsubsetneqq F_{2}$. $D$ is a maximal element of $\Sigma$, so $F_{1} \cap I \neq \emptyset$ and $F_{2} \cap I \neq \emptyset$. Therefore $\exists a_{1} \in F_{1} \cap I$ and $\exists b_{1} \in F_{2} \cap I$, so there exist $d_{1}, d_{2} \in D$, such that $a_{1} \in d_{1} \wedge a^{\prime}, a^{\prime} \geq a$ and $b_{1} \in d_{2} \wedge b^{\prime}, b^{\prime} \geq b$. We claim that $a_{1} \vee b_{1} \in D \cap I$, which is a contradiction.

By $a^{\prime} \geq a$ and $b^{\prime} \geq b$, we have $a \vee b \leq a^{\prime} \vee b^{\prime}$ and since $a \vee b \in D$ and $D$ is a hyperfilter, $a^{\prime} \vee b^{\prime} \in D$.

Since $a_{1}, b_{1} \in I$ and $I$ is a hyperideal, $a_{1} \vee b_{1} \in I$. Also we have $a_{1} \vee b_{1} \in\left(d_{1} \wedge a^{\prime}\right) \vee\left(d_{2} \wedge b^{\prime}\right)$. $L$ is distributive, so we have:

$\left(d_{1} \wedge a^{\prime}\right) \vee\left(d_{2} \wedge b^{\prime}\right)=\left(d_{2} \vee d_{1}\right) \wedge\left(d_{2} \vee a^{\prime}\right) \wedge\left(b^{\prime} \vee d_{1}\right) \wedge\left(b^{\prime} \vee a^{\prime}\right) \in D$. So $a_{1} \vee b_{1} \in D$. Thus $a_{1} \vee b_{1} \in D \cap I$.

Corollary 63. (i) Let $L$ be distributive, and $a \in L$. If $F$ is a hyperfilter of $L$, such that $a \notin F$, then there exists prime hyperfilter $D$, such that $F \subseteq D$, and $a \notin D$.

(ii) Let $L$ be a distributive " $\wedge "$-hyperlattice, and $a, b \in L$. If $a \neq b$, then there exists prime hyperfilter $D$ of $L$, such that $a \in D$, and $b \notin D$.

Theorem 64 (Prime Hyperideal Theorem). Let $L$ be dual distributive. If I is a hyperideal and $F$ is a hyperfilter of $L$, such that $I \cap F=\emptyset$, then there exists prime hyperideal $P$ of $L$, such that $I \subseteq P$ and $P \cap F=\emptyset$.

Definition 65. Let $L$ be a bounded $\wedge$-hyperlattice. Then: 
M. Amiri Bideshki, R. Ameri, A. Borumand Saeid / Eur. J. Pure Appl. Math, 11 (1) (2018), 169-188 184

(i) $x^{\prime} \in L$ is called a complement of $x \in L$, if $0 \in x \wedge x^{\prime}$ and $x \vee x^{\prime}=1$. Also, we say that $x \in L$ has a complement if there exists $x^{\prime} \in L$ such that $0 \in x \wedge x^{\prime}$ and $x \vee x^{\prime}=1$. Also we say that the $\wedge$-hyperlattice $L$ is complemented if every element $x \in L$ has a complement.

(ii) $L$ is called a good complemented $\wedge$-hyperlattice, if for all $x \in L$, there exists $x^{\prime} \in L$ such that $x \wedge x^{\prime}=\{0\}$ and $x \vee x^{\prime}=1$

Example 66. Let $L=\{0, x, y, z, 1\}$. Define $\wedge$ and $\vee$ with tables 6 Then $(L, \wedge, \vee)$ is a

\begin{tabular}{c|ccccc}
$\wedge$ & 0 & $x$ & $y$ & $z$ & 1 \\
\hline 0 & $\{0\}$ & $\{0\}$ & $\{0\}$ & $\{0\}$ & $\{0\}$ \\
$x$ & $\{0\}$ & $\{0, x\}$ & $\{0, x\}$ & $\{0\}$ & $\{0, x\}$ \\
$y$ & $\{0\}$ & $\{0, x\}$ & $\{y\}$ & $\{0\}$ & $\{y\}$ \\
$z$ & $\{0\}$ & $\{0\}$ & $\{0\}$ & $\{z\}$ & $\{z\}$ \\
1 & $\{0\}$ & $\{0, x\}$ & $\{y\}$ & $\{z\}$ & $\{1\}$
\end{tabular}

(a)

\begin{tabular}{l|lllll}
$\vee$ & 0 & $x$ & $y$ & $z$ & 1 \\
\hline 0 & 0 & $x$ & $y$ & $z$ & 1 \\
$x$ & $x$ & $x$ & $y$ & 1 & 1 \\
$y$ & $y$ & $y$ & $y$ & 1 & 1 \\
1 & 1 & 1 & 1 & 1 & 1
\end{tabular}

(b)

Table 6

$\wedge$-hyperlattice; We have $0 \in y \wedge z$ and $y \vee z=1$ also, $0 \in x \wedge z$ and $x \vee z=1$, so $y, x$ are complement of $z$. Also $L$ is a good complemented $\wedge$-hyperlattice.

Proposition 67. Let $L$ be a bounded strongly distributive $\wedge$-hyperlattice. Then the following conditions hold.

(i) If $a, b$ are complement of $x$, then $a \vee b$ is so.

(ii) If $a, b$ are complements of $x$ and $x \wedge x=x$, then there exists $c, d \in a \wedge b$ such that $1=x \vee c$ and $0 \in x \wedge d$.

Proof. (i): We have $0 \in x \wedge a$ and $x \vee a=1$, also $0 \in x \wedge b$ and $x \vee b=1$. Since $0 \wedge 0=0$ and $L$ is dual distributive, we have:

$0 \in(x \wedge a) \vee(x \wedge b)=x \wedge(a \vee b)$, thus $0 \in x \wedge(a \vee b)$. Also $1=1 \vee 1$ and we have:

$1=1 \vee 1=(x \vee a) \vee(x \vee b)=x \vee(a \vee b)$, thus $x \vee(a \vee b)=1$. So $a \vee b$ is a complement of $x$.

(ii):Since $1 \in 1 \wedge 1,0 \vee 0=0$, and $L$ is strongly distributive, we have: $1 \in 1 \wedge 1=(x \vee a) \wedge(x \vee b)=x \vee(a \wedge b)$, so there exists $c \in a \wedge b$, such that $1=x \vee c$. $0 \in 0 \wedge 0 \subseteq(x \wedge a) \wedge(x \wedge b)=(x \wedge x) \wedge(a \wedge b)=x \wedge(a \wedge b)$; So, there exists $d \in a \wedge b$ such that $0 \in x \wedge d$.

Lemma 68. (Demorgan Laws) Let $L$ be a good complemented and strongly distributive $" \wedge "$-hyperlattice. Then the following statements for all $x, y \in L$, hold.

(i) $(x \vee y)^{\prime} \in x^{\prime} \wedge y^{\prime}$. 
M. Amiri Bideshki, R. Ameri, A. Borumand Saeid / Eur. J. Pure Appl. Math, 11 (1) (2018), 169-188 185

(ii) $x^{\prime} \vee y^{\prime} \in(x \wedge y)^{\prime}$.

Example 69. Let $L=\{0, x, y, z, 1\}$. $\wedge$-hyperoperation and $\vee$-operation are given by Tables7. We have $(y \vee 0)^{\prime} \neq y^{\prime} \wedge 0^{\prime}$, because $(y \vee 0)^{\prime}=y^{\prime}=z$ and $y^{\prime} \wedge 0^{\prime}=\{0, z\}$. Thus $(y \vee 0)^{\prime} \varsubsetneqq y^{\prime} \wedge 0^{\prime}$. Also In this example, every of $x, y, z$ has two complements. We have

\begin{tabular}{c|ccccc}
$\wedge$ & 0 & $x$ & $y$ & $z$ & 1 \\
\hline 0 & 0 & 0 & 0 & 0 & 0 \\
$x$ & 0 & $\{0, x\}$ & 0 & 0 & $\{0, x\}$ \\
$y$ & 0 & 0 & $\{0, y\}$ & 0 & $\{0, y\}$ \\
$z$ & 0 & 0 & 0 & $\{0, z\}$ & $\{0, z\}$ \\
1 & 0 & $\{0, x\}$ & $\{0, y\}$ & $\{0, z\}$ & $L$
\end{tabular}

(a)

\begin{tabular}{c|ccccc}
$\vee$ & 0 & $x$ & $y$ & $z$ & 1 \\
\hline 0 & 0 & $x$ & $y$ & $z$ & 1 \\
$x$ & $x$ & $x$ & 1 & 1 & 1 \\
$y$ & $y$ & 1 & $y$ & 1 & 1 \\
$z$ & $z$ & 1 & 1 & $z$ & 1 \\
1 & 1 & 1 & 1 & 1 & 1
\end{tabular}

(b)

Table 7

$x^{\prime}=\{y, z\}, y^{\prime}=\{x, z\}, z^{\prime}=\{x, y\}$.

It is obvious that if complement of $x$ is unique, then $x=x^{\prime \prime}$. $x$ and its complemet are not comparable where $x \neq 0,1$.

Corollary 70. Let L be a good complemented and strongly distributive" $\wedge$ "-hyperlattice. If $x$ is a complement of $y$ then we have:

(i) $(x \vee y)^{\prime}=x^{\prime} \wedge y^{\prime}$;

(ii) $(x \wedge y)^{\prime}=x^{\prime} \vee y^{\prime}$.

Theorem 71. Let $L$ be a distributive and good complemented $\wedge$-hyperlattice. Then we have:

(i) Complement of any element of $L$ is unique.

(ii) If $x, y \in L$ such that $x \leq y$, then $y^{\prime} \leq x^{\prime}$.

Proof. (i): Let $a, b$ be complements of $x$. Then $b \wedge x=\{0\}$ and we have:

$$
a=a \vee 0=a \vee(b \wedge x)=(a \vee b) \wedge(a \vee x)=(a \vee b) \wedge 1
$$

Since $a \vee b \leq 1, a \vee b \in(a \vee b) \wedge 1$. So $a \vee b=a$ and it implies that $b \leq a$, similarly, it is proved that $a \leq b$; So $a=b$.

(ii): Since $x \leq y, x \vee y=y$. So $y^{\prime}=(x \vee y)^{\prime}$. By Demorgan law, We have $(x \vee y)^{\prime} \in x^{\prime} \wedge y^{\prime}$, then $y^{\prime} \in x^{\prime} \wedge y^{\prime}$, and it implies that $y^{\prime} \leq x^{\prime}$.

Remark 72. In Theorem 71, if $L$ is a dual distributive good complemented" $\wedge$ "-hyperlattice, then $(i)$ holds. Since $a \leq 1$, We have: $a \in a \wedge 1=a \wedge(b \vee x)=(a \wedge b) \vee(a \wedge x)=(a \wedge b) \vee 0=$ $a \wedge b$, so $a \in a \wedge b$ and it implies that $a \leq b$. Similarly, it is proved that $b \leq a$. So $a=b$. 
Theorem 73. Let $P$ be a proper hyperfilter of a complemented" $\wedge$ "-hyperlattice L. Then $P$ is a prime hyperfilter, if and only if $x \vee y^{\prime} \in P$ and $y \vee x^{\prime} \in P$, where $x, y \in L \backslash P$,

Example 74. Consider" $\wedge "$-hyperlattice $L$ in Example 66. Then $\{z, 1\}$ and $\{0, x, y, 1\}$ are two prime hyperfilters of $L . F=\{y, 1\}$ is a hyperfilter of $L$, but it is not an prime hyperfilter, because of $x, z \notin F$, but $x^{\prime} \vee z=z \vee z=z \notin F$.

Theorem 75. Let $P$ be a proper hyperfilter of a good complemented" $\wedge$ "-hyperlattice $L$. Then $P$ is a prime hyperfilter of $L$ if and only if $x \in P$ or $x^{\prime} \in P, \forall x \in L \quad(x \in P$ iff $\left.x^{\prime} \notin P\right)$.

Proof. Let $P$ be a prime hyperfilter and there exists $x \in L$ such that $x \notin P$ and $x^{\prime} \notin P$. By Theorem 73, $x \vee x^{\prime \prime} \in P$ and $x^{\prime} \vee x^{\prime} \in P$. Therefore $x \in P$ and $x^{\prime} \in P$, which is a contradiction.

Conversely, let $x \in P$ or $x^{\prime} \in P$, for all $x \in L$. Suppose that $x, y \notin P$. If $x \vee y^{\prime} \notin P$ or $y \vee x^{\prime} \notin P$, then $\left(x \vee y^{\prime}\right)^{\prime} \in P$ or $\left(y \vee x^{\prime}\right)^{\prime} \in P$. We have $y^{\prime} \leq x \vee y^{\prime}$, by Theorem 71, $\left(x \vee y^{\prime}\right)^{\prime} \leq y$. Since $P$ is a hyperfilter and $\left(x \vee y^{\prime}\right)^{\prime} \in P, y \in P$, which is a contradiction. If $\left(y \vee x^{\prime}\right)^{\prime} \in P$, similarly, it is proved that $x \in P$, which is a contradiction.

Theorem 76. Let $L$ be a complemented $\wedge$-hyperlattice. Then every prime hyperfilter of $L$ is a maximal hyperfilter.

Proof. Let $P$ be a prime hyperfilter and $F$ be a hyperfilter of $L$ such that $P \varsubsetneqq F$. So there exists $a \in F \backslash P$, since $P$ is a prime hyperfilter and $a \notin P, a^{\prime} \in P \subseteq F$. We have $a, a^{\prime} \in F$; Since $F$ is a hyperfilter, $x \wedge x^{\prime} \subseteq F$. So $0 \in F$, since $0 \leq x$ for all $x \in L, x \in F$. Then $F=L$, and we conclude that $P$ is a maximal hyperfilter.

Problem: Under what suitable condition the converse of Theorem76 is correct?

Theorem 77. Let $L$ be a distributive good complemented $\wedge$-hyperlattice. Then the following conditions are equivalent.

(i) $\{1\}$ is a prime hyperfilter of $L$.

(ii) all the hyperfilters of $L$ are prime.

(iii) $(L, \leq)$ is a chain and $L=\{0,1\}$.

Proof. $(i) \Longrightarrow($ ii $)$ Let $F$ be a filter of $L$. Assume that $x \in L$ and $x \notin F$. We must show that $x^{\prime} \in F$. Since $x \vee x^{\prime}=1$ and $\{1\}$ is a prime filter, so $x=1$ or $x^{\prime}=1$. We know that $x \neq 1$, thus $x^{\prime}=1 \in F$.

$(i i) \Longrightarrow(i)$ is obvious.

$(i) \Longrightarrow$ (iii) Since $\{1\}$ is a prime hyperfilter of $L, \forall x \in L, x=1$ or $x^{\prime}=1$. We have $x \neq 1$, also since $L$ is distributive good complemented and $x^{\prime}=1$, by Theorem 71 , we conclude that $x=0$. Therefore $L=\{0,1\}$ and $L$ is a chain.

$($ iii $) \Longrightarrow(i)$ is obvious. 


\section{References}

[1] R. Ameri and T. Nozari, "A Connection Between Categories of Multialgebras and Algebra", Italian Journal of Pure and Applied Mathematics, Vol. 27, 201-208, 2010.

[2] R. Ameri and I. G. Rosenberg, "Congruences of Multialgebras", J. of Multi-Valued Logic \& Soft Computing, Vol. 00, 1-12, 2009.

[3] R. Ameri and M. M. Zahedi, "Hyperalgebraic System", Italian Journal of Pure and Applied Mathematics, Vol. 6, 21-39, 1999.

[4] A. Asokkumar, "Hyperlattice Formed by the Idempotents of a Hyperring", International Journal of Mathematics, Vol. 38, 209-215, 2007.

[5] G. E. Hansoul, "A Simultianeous Characterization of Subalgebras and Conditional Sunalgebras of a Multialgebra", Bull. Soc. Roy. Science Liego, Vol. 50, 16-19, 1981.

[6] P. He, X. Xin, and Jianming Zhan, "On Rough Hyperideals in Hyperlattices", Journal of Applied and Mathematics, Vol. 2013, 10 Page.

[7] B. B. N. Koguep, C. Nkuimi, C. Lele, "On Fuzzy Ideals of Hyperlattice," International Journal of Algebra, Vol. 2, 739-750. 2008.

[8] M. Konstantinidou-Serafimidou, "Modular Hyperlattices", $\Gamma$ Ranktika tes Akademias Athenon, Vol. 53, 202-218, 1978.

[9] M. Konstantinidou-Serafimidou, "Distributive and Complemented Hyperlattices", PraKtika Tes Akademias Athenon, Vol. 56, 339-360, 1981.

[10] M. Konstantinidou, J. Mittas, "An introduction to the Theory of Hyperlattice," Math. Balcanica, Vol. 7, 187- 193, 1977.

[11] F. Marty, "Surene generalization de la notion de group, In Eighth Congress Scandinaves," Stockholm, 45-49, 1934.

[12] G. A. Moghani, A. R, Ashrafi, "On Some Hypergroups and Their Hyperlattice Structures", Buletinul Academiei De Stiinte, Vol. 3, 15-24, 2003.

[13] C. Pelea, "Multialgebras, Universal Algebra, and Identities", J. Aust. Math, Vol. 81, 121-139, 2006.

[14] C. Pelea, "On the Direct Limit of a Direct System of Multialgebras",Direct Mathematics, Vol. 306, 2916-2930, 2006.

[15] C. Pelea, "Hyperring and $\alpha^{*}$ - Relations. A General Approach", Journal of Algebra, Vol. 383, 104-128, 2013.

[16] C. Pelea and I. Purdea, "A Characterization Theorem For Complete Multialgebras", Mathematica, 205-211, 2004. 
[17] H. E. Pickett, "Subdirect Representations of Related System", Fund. Math, Vol. 56, 223-240, 1964.

[18] H. E. Pickett, "Homomorphisms and Subalgebras of Multialgebras", Pacific J. of Math, Vol. 21, 327-343, 1967.

[19] A. Rahnemai-Barghi, "The Prime Ideal Theorem for Distributive Hyperlattices," Ital. J. Pure Appl. Math. , Vol. 10, 75-78, 2001.

[20] S. Rasouli, B. Davvaz, "Lattice Derived from Hyperlattices", Communications in Algebra, Vol. 38, 2720- 2737, 2010.

[21] S. Rasouli, B. Davvaz, "Construction and Spectral Topology on Hyperlattice", Mediterr. J. Math., Vol. 7, 249- 262, 2010.

[22] D. Schweigrt, "Congruence Relation of multialgebra", Discrete Mathematics", Vol. 53, 249-253, 1985.

[23] X. L. Xin and X. G. Li, "On Hyperlattice and Quotient Hyperlattice", 